\title{
Microbiota of the prostate tumor environment investigated by whole- transcriptome profiling
}

\author{
Paul Vinu Salachan ${ }^{1,2}$, Martin Rasmussen ${ }^{1,2}$, Jacob Fredsøe ${ }^{1,2}$, Benedicte Ulhøi ${ }^{3}$, Michael Borre ${ }^{2,4}$ and
} Karina Dalsgaard Sørensen ${ }^{1,2^{*}}$ (D)

\begin{abstract}
Background: With over 350,000 estimated deaths worldwide in 2018, prostate cancer (PCa) continues to be a major health concern and a significant cause of cancer-associated mortality among men. While cancer in general is considered a disease of the human genome, there is a growing body of evidence suggesting that changes to the healthy microbiota could play a vital role in cancer development, progression, and/or treatment outcome.

Methods: Using a metatranscriptomic approach, we annotated the microbial reads obtained from total RNA sequencing of 106 prostate tissue samples from 94 PCa patients (discovery cohort). We investigated microbial dysbiosis associated with PCa by systematically comparing the microbiomes between benign and malignant tissue samples, between less vs. more-aggressive PCa, and between patients who had biochemical recurrence as opposed to those who did not. We further performed differential gene expression and cell type enrichment analysis to explore the host transcriptomic and cellular responses to selected microbial genera. A public dataset (GSE115414) of total RNA sequencing reads from 24 prostate tissue samples (8 benign and 16 malignant) served as the validation cohort.

Results: We observed decreased species diversity and significant under-representation of Staphylococcus saprophyticus and Vibrio parahaemolyticus, as well as significant over-abundance of Shewanella in malignant as compared to benign prostate tissue samples in both the discovery $(p<0.01)$ and validation $(p<0.05)$ cohorts. In addition, we identified Microbacterium species $(p<0.01)$ to be significantly over-abundant in pathologically advanced T3 tumors compared to T2 in the discovery cohort. Malignant samples having high vs. low Shewanella counts were associated with downregulated Toll-like receptor signaling pathways and decreased enrichment of dendritic cells. Malignant samples having low vs. high V. parahaemolyticus counts were enriched for olfactory transduction and drug metabolism pathways. Finally, malignant samples were enriched for M1 and M2 macrophages as compared to benign tissue samples.
\end{abstract}

\footnotetext{
* Correspondence: kdso@clin.au.dk

'Department of Molecular Medicine, Aarhus University Hospital, 8200 Aarhus

N, Denmark

${ }^{2}$ Department of Clinical Medicine, Aarhus University, 8200 Aarhus N,

Denmark

Full list of author information is available at the end of the article
}

(c) The Author(s). 2022 Open Access This article is licensed under a Creative Commons Attribution 4.0 International License, which permits use, sharing, adaptation, distribution and reproduction in any medium or format, as long as you give appropriate credit to the original author(s) and the source, provide a link to the Creative Commons licence, and indicate if changes were made. The images or other third party material in this article are included in the article's Creative Commons licence, unless indicated otherwise in a credit line to the material. If material is not included in the article's Creative Commons licence and your intended use is not permitted by statutory regulation or exceeds the permitted use, you will need to obtain permission directly from the copyright holder. To view a copy of this licence, visit http://creativecommons.org/licenses/by/4.0/. The Creative Commons Public Domain Dedication waiver (http://creativecommons.org/publicdomain/zero/1.0/) applies to the data made available in this article, unless otherwise stated in a credit line to the data. 
Conclusions: The results from this exploratory study support the existence of an important biological link between the prostate microbiota and PCa development/progression. Our results highlight Shewanella, V. parahaemolyticus, and Microbacterium sp. as interesting candidates for further investigation of their association with PCa.

Keywords: Prostate cancer, Microbiome, Metatranscriptome, Vibrio parahaemolyticus, Microbacterium sp., Shewanella, Dendritic cells

\section{Background}

Prostate cancer $(\mathrm{PCa})$ remains the most prevalent cancer among men in Denmark and the second most incident cancer among men worldwide [1]. While mortality rates associated with $\mathrm{PCa}$ have shown a declining trend in some European countries [2], PCa still contributes to a significant fraction of global cancer mortality among men [1] with a continued rise globally due to population growth and increasing population age [1,2].

While major risk factors for $\mathrm{PCa}$ include ethnicity, age, genetic predisposition, and a family history of PCa [3, 4], the role of inflammation in relation to PCa development and progression has also garnered scientific attention (reviewed in [5, 6]). Given the intricate relationship between inflammation and the host microbiota [7], the human microbial ecosystem is increasingly being implicated in the occurrence of various cancers with causal relationships reported in a few instances, e.g., Helicobacter pylori infection and gastric carcinoma [8].

Cancer tissue may also harbor unique microbial signatures that could be of diagnostic, prognostic, and/or therapeutic potential. For example, a recent large-scale study [9] found microbial signatures in tumors in several cancer types (e.g., stomach and lung adenocarcinomas) that were unique to each. Few earlier and smaller-scale studies specifically investigating microbial signatures in benign and malignant prostate tissue have also found some evidence to support a PCa-specific microbiota [10, 11]. However, other small-scale studies have failed to clearly discriminate benign vs. malignant prostate tissues based on either metagenomic or metatranscriptomic data [12], suggesting considerable variation in the presence of microbial signatures between patient cohorts. For example, it has been reported that prostate tumor tissue from African patients might harbor different microbial loads compared to Australian patients [13]. Nevertheless, all these studies support the existence of a non-sterile prostate microenvironment.

Currently, although no single pathogenic species has been implicated in PCa development, it is believed that microbial dysbiosis (i.e., changes to the healthy microbiota) could play a significant role in disease occurrence, progression, and/or treatment outcome [14], even though this area is still understudied. Hence, in order to study any potential dysbiosis associated with PCa development and progression, we analyzed the microbiome of benign (adjacent normal) and malignant (tumor) prostate tissue samples from a total of 94 Danish men with PCa. Using total RNA sequencing (RNAseq), we obtained and annotated microbial reads from all samples and systematically compared the microbiomes between benign and malignant tumor tissue, less vs. moreaggressive $\mathrm{PCa}$, and between patients who had biochemical recurrence (BCR) as opposed to those who did not (BCR-free). We further characterized the host transcriptional regulation in response to increased/decreased abundance of specific organisms within the malignant tissue samples and subsequently performed cell type enrichment to explore differences in cellular composition within malignant tissue samples having high vs. low count of specific organisms.

\section{Methods \\ Patient cohorts}

Our PCa discovery cohort consisted of 114 prostate tissue samples from 102 patients who underwent curatively intended radical prostatectomy (RP) for histologically verified, clinically localized $\mathrm{PCa}$, at the Department of Urology, Aarhus University Hospital (AUH), Denmark (2004-2017). Immediately following surgery, fresh tissue biopsies were obtained and stored at $-80^{\circ} \mathrm{C}$ in TissueTek until further processing. Prior to RNA extraction, all tissue specimens were marked as either benign or malignant $(\mathrm{PCa})$ based on histopathological examination of the H\&E stained tissue sections by an experienced pathologist at AUH. Eight malignant tissue samples were excluded due to very high unmapped read counts, contributed primarily by known contaminant taxa [15]. Thus, the final cohort consisted of 106 tissue samples (23 benign and 83 malignant) from 94 patients (Additional file 1: Fig. S1). Clinical follow-up for this cohort was updated in November 2019.

For validation, we used a publically available dataset of total RNA sequencing data from a small cohort of PCa patients recruited in France [16, 17]. Collection of benign and tumor biopsy specimens was done retrospectively from the patients who gave informed consent. The cohort consisted of 24 prostate tissue samples ( 8 benign and 16 malignant) from men who underwent radical prostatectomy (Additional file 1: Fig. S1). Formalin-fixed paraffin-embedded tissue samples were used for total RNA extraction and sequencing. Clinicopathological 
characteristics for the discovery cohort and the validation cohort are given in Table 1.

\section{Total RNA extraction and sequencing}

For the discovery cohort, total RNA was extracted from fresh-frozen prostate tissue samples using the RNeasy Plus Mini Kit (Qiagen). The concentration and quality of RNA was assessed using a NanoQuant and an Agilent 2100 Bioanalyzer ( $\mathrm{RIN} \geq 7$ ), respectively. Sequencing libraries were generated using either the ScriptSeq RNA-seq Library Kit with the Ribo-Zero ${ }^{\text {tm }}$ Magnetic Gold Kit from Illumina (37 malignant and 5 benign samples) or the KAPA RNA HyperPrep Kit with KAPA RiboErase Kit from Roche (46 malignant and 18 benign samples). All libraries were sequenced using Illumina NovaSeq or NextSeq 500. All reads were $\mathrm{QC}$ checked and aligned to the human reference genome hg38 using STAR [18]. On average, each sample bam file contained approximately 76 million 75bp reads (38 million read pairs) and approximately 60 million reads per sample aligned to hg38. We obtained approx. 1 million read pairs per sample that had both the mate pairs unmapped which we used for microbial read processing (Table 1).

For the validation cohort [16], we downloaded a public dataset of total RNA sequencing reads from the gene expression omnibus portal (accession number GSE115414 [17]). Alignment of the reads was performed as described above. On average, each sample bam file contained approximately 300 million reads and approximately 170 million reads per sample aligned to hg38. We obtained approx. 2.7 million read pairs per sample that had both the mate pairs unmapped which we used for microbial read processing (Table 1 ).

Table 1 Clinicopathological and sequencing characteristics

\begin{tabular}{|c|c|c|}
\hline \multicolumn{3}{|l|}{ Clinicopathological characteristics } \\
\hline Characteristic & Discovery cohort & Validation cohort \\
\hline Patients, N & 94 & 24 \\
\hline Tissue samples, $N$ & 106 & 24 \\
\hline Age at RP, median (range) & $65.7(45.7-76.6)$ & N/A \\
\hline \multicolumn{3}{|l|}{ Tumor status } \\
\hline Benign, N (\%) & $23(21.7 \%)$ & $8(33.3 \%)$ \\
\hline Malignant, N (\%) & $83(78.3 \%)$ & $16(66.6 \%)$ \\
\hline \multicolumn{3}{|l|}{ Pathological Gleason score } \\
\hline$\leq 7, N(\%)$ & $63(75.9 \%)$ & $9(56.2)$ \\
\hline$>7, N(\%)$ & $20(24.1 \%)$ & $7(43.7)$ \\
\hline \multicolumn{3}{|l|}{ Pre-operative PSA (ng/ml) } \\
\hline$\leq 10, N(\%)$ & $39(47 \%)$ & N/A \\
\hline$>10, N(\%)$ & $39(47 \%)$ & N/A \\
\hline Unknown, N (\%) & $5(6 \%)$ & N/A \\
\hline \multicolumn{3}{|l|}{ Pathological T-stage } \\
\hline $\mathrm{pT} 2, N(\%)$ & $51(61.4 \%)$ & $4(25 \%)$ \\
\hline pT3, N (\%) & $31(37.4 \%)$ & $10(62.5 \%)$ \\
\hline Unknown/pT4, N (\%) & $1(1.2 \%)$ & $2(12.5 \%)$ \\
\hline \multicolumn{3}{|l|}{ Biochemical recurrence status } \\
\hline $\mathrm{BCR}, N(\%)$ & $28(33.7 \%)$ & $6(37.5 \%)$ \\
\hline BCR-free, $N(\%)$ & $53(63.9 \%)$ & $10(62.5 \%)$ \\
\hline Unknown, N (\%) & $2(2.4 \%)$ & -- \\
\hline Median follow-up, months (range) & $52.4(17.6-178.9)$ & N/A \\
\hline \multicolumn{3}{|l|}{ Sequencing characteristics } \\
\hline Characteristic & Discovery Cohort & Validation cohort \\
\hline Total reads in bam per sample, mean (SD) & $76,688,094(11,296,752)$ & $304,920,301(49,502,192)$ \\
\hline Reads per sample mapped to hg38, mean (SD) & $60,131,687(7,869,239)$ & $170,390,820(16,853,739)$ \\
\hline Unmapped read pairs per sample, mean (SD) & $970,526(280,812)$ & $2,734,147(421,428)$ \\
\hline
\end{tabular}

$R P$ radical prostatectomy, $P S A$ prostate-specific antigen, $S D$ standard deviation, $\mathrm{Hg}$ human genome 


\section{Host read processing}

Host transcript levels were quantified using kallisto [19] with hg38 as the reference transcriptome. Transcripts were aggregated to gene level using tximport [20]. Batch effects were either adjusted for in the design formula (for differential gene expression analyses) or removed using the RemoveBatchEffect function (for cell type enrichment analyses) within Limma [21].

\section{Microbial read processing}

We followed a modified version of the SAMSA2 pipeline [22] for microbial read processing and differential microbial abundance analysis. Briefly, fastq files of reads that did not map to the human reference genome hg38 were generated from bam files using BEDTools [23] and thereafter paired-end reads were merged using PEAR [24]. DIAMOND [25] sequence aligner was used to align and annotate the unmapped reads against NCBI RefSeq bacterial non-redundant protein sequences database [26]. For annotation against viral sequences, additional viral protein sequences were downloaded from NCBI RefSeq database [26]. Custom python and $\mathrm{R}$ scripts provided with the SAMSA2 pipeline [22] were used for aggregating and merging the annotation files. The aggregated files with microbial read counts were subsequently used for downstream analysis.

\section{Comparison of overall microbial diversity}

For both cohorts, we compared the microbial species diversity within malignant and benign prostate tissue samples by estimating the alpha diversity as implemented in the $\mathrm{R}$ phyloseq [27] and vegan [28] packages. Alpha diversity measures the total number of species (i.e., species richness) and their relative proportions in a population (i.e., evenness). Accordingly, differences in alpha diversities between malignant and benign prostate tissue samples would signify microbial dysbiosis and could be associated with disease occurrence. For visualizing alpha diversity, we used six different measures: Observed, Chao1, and ACE capture aspects of species richness, whereas Shannon, Simpson, and Inverse Simpson capture both species richness and evenness [29-31]. A Wilcoxon rank sum test was used to compare each diversity estimate between the two groups. $P$ values were corrected for family wise error rate using the Bonferroni method [32] and significance was determined at a $p$ value cut-off less than 0.05 . We consider differences in alpha diversity between groups to be reliable only if the Shannon, Simpson, and Inverse Simpson diversity estimates showed a significant difference as these are more robust measures of alpha diversity [33].

In addition, alpha diversity was also estimated for the samples in the comparisons between low vs. high Gleason scores, low vs. high pre-operative Prostate-Specific
Antigen (pre-op PSA) levels, pathological T-stage 2 vs. 3 , and BCR vs. BCR-free groups for the discovery cohort (Additional file 1: Fig. S1). Due to the limited sample size, we did not include these analyses for the validation cohort.

\section{Differential microbial abundance analysis}

Differential abundances between malignant $(n=83)$ and benign prostate tissue samples $(n=23)$ of microbial counts (as well as for other comparisons) in the discovery cohort were tested using the DESeq2 package [34] in $\mathrm{R}$, and $p$ values were adjusted for multiple testing using Benjamini-Hochberg [35] corrections. We used an adjusted $p$ value less than 0.01 to make statistical inferences, as this would allow us to make five comparisons if the original significance level was at 0.05 (accounting for family-wise error rate from multiple comparisons made on the same dataset). Further, species having a $p$ value less than 0.01 were considered to be significantly over-represented if they had a $\log _{2}$ fold change greater than 0.58 (corresponding to a fold change of 1.5) in the given comparison and under-represented if the $\log _{2}$ fold change was less than -0.58 in any given comparison. In order to avoid potential biases from species with low read counts, we filtered out the low abundance species having a mean normalized count of fewer than 10 across all samples. Since our samples were prepared using two different RNAseq library preparation kits, we accounted for batch effects in the differential abundance analysis by including the batch factor in the design formula.

In order to investigate microbial dysbiosis in less vs. more-aggressive $\mathrm{PCa}$, we systematically compared malignant tissue samples from patients having low $(\leq 7, n=$ $63)$ vs. high $(>7, n=20)$ Gleason scores, low ( $\leq 10 \mathrm{ng} /$ $\mathrm{ml}, n=39$ ) vs. high ( $>10 \mathrm{ng} / \mathrm{ml}, n=39$ ) pre-operative Prostate-Specific Antigen (pre-op PSA) levels, and a pathological T-stage $2(\mathrm{pT} 2, n=51)$ vs. $3(\mathrm{pT} 3, n=31)$. We further looked for differences in microbial abundance in patients who had BCR $(n=28)$ after RP compared to those who did not (BCR-free, $n=53$ ). Postoperative BCR was defined as a PSA $\geq 0.2 \mathrm{ng} / \mathrm{ml}$. Patients not suffering BCR were censored at their last normal PSA measurement. An overview of the different comparisons is given in Additional file 1: Fig. S1.

To validate the differentially abundant organisms identified between malignant and benign prostate tissue samples in the discovery cohort, we compared their differential abundance between malignant $(n=16)$ and benign prostate tissue samples $(n=8)$ in an independent set of samples (validation cohort). Due to the limited sample size (and composition) in this validation cohort, we could not test for differential abundance between less vs. more-aggressive PCa. 


\section{Differential host transcriptional regulation}

For the species identified as differentially over/less abundant in the malignant as compared to the benign tissue samples in both the cohorts, we performed host differential gene expression (DGE) analyses for elucidating the host transcriptional regulation in response to high $(n=$ $42)$ or low $(n=41)$ species counts. Low species count was defined as a mean normalized count less than the median count. DGE analyses were performed similar to the methodology described above using the DESeq2 package [34] in R, however, with a significance cut-off of adjusted $p$ value less than 0.05 . Genes identified as differentially upregulated or downregulated were each used for Kyoto Encyclopedia of Genes and Genomes (KEGG) pathway analyses using DAVID bioinformatics resources [36]. A false discovery rate (FDR) less than 0.05 was used as a cut-off for determining significant pathways.

\section{Cell type enrichment analyses}

For determining the cellular composition of the malignant tissue samples harbouring high $(n=42)$ or low $(n$ $=41$ ) counts of the species identified as either differentially over-abundant or differentially less abundant in the malignant samples in both the cohorts, we performed cell type enrichment analyses using xCell [37] in R for the tissue samples in the discovery cohort. Using this approach, we explored the stromal, epithelial, and immune cell types within the malignant tissue samples. Associations between the species abundance and the host cell types were tested using a Wilcoxon test with an FDR cut-off less than 0.05 used for determining significant associations.

Similarly, we also explored the host cellular composition between benign $(n=23)$ and malignant $(n=83)$ tissue samples using $\mathrm{xCell}$, in order to find associations between specific immune/stromal cell types and PCa, which in turn may be linked with microbial dysbiosis in PCa.

\section{PCR validation of selected species}

To validate the presence of microbial species within tissue samples, we selected Bacteroides fragilis as a candidate organism, due to its frequent presence in prostate tissue samples as identified from our microbiome analysis. Primers specific to $B$. fragilis were selected from the literature [38]. RNA extracted from 6 fresh-frozen RP tissue samples ( 3 malignant and 3 benign) was reverse transcribed using a mix of oligo $\mathrm{dT}$ and random hexamer primers. cDNA was amplified by PCR with a total reaction volume of $15 \mu \mathrm{l}$ as follows: initial denaturation at $95^{\circ} \mathrm{C}$ for $5 \mathrm{~min}$ followed by 35 cycles of $94^{\circ} \mathrm{C}$ for $30 \mathrm{~s}, 50^{\circ} \mathrm{C}$ for $1 \mathrm{~min}$, and $72^{\circ} \mathrm{C}$ for $1 \mathrm{~min}$. A final extension was performed at $72^{\circ} \mathrm{C}$ for $10 \mathrm{~min}$. The PCR product was run on a $1 \%$ agarose gel, and the presence of a 495-bp amplification product was verified using a gel doc system. Next, $10 \mu$ PCR product was used for PCR clean-up and subsequent Sanger sequencing to verify the B. fragilis sequence. Forward and reverse reads from the sequencing were assembled into contigs using GeneStudio. Local alignment search against 16s ribosomal RNA sequences was performed using NCBI BLAST [39] optimized to retrieve highly similar sequences (megablast). Uncultured and environmental sample sequences were excluded from the search.

\section{Results}

For metatranscriptomic analyses, we used a set of total RNA sequencing data from benign and malignant prostate tissue samples, annotated against known microbial reads in NCBI. The dataset included 106 tissue samples from 94 PCa patients (discovery cohort, Additional file 1: Fig. S1). The median patient age at RP was 65.7 years. Almost $76 \%$ of the tissue samples had a Gleason score $\leq$ 7 and $24 \%$ had a score greater than 7 . An equal representation of samples had a pre-op PSA value $\leq 10(47 \%)$ and $>10 \mathrm{ng} / \mathrm{ml}(47 \%)$, whereas approx. $61 \%$ and $37 \%$ of the samples had a pathological T-stage of pT2 and pT3, respectively. Almost $64 \%$ of the samples were from patients without BCR, while approx. $34 \%$ experienced BCR (median follow-up of 52.4 months). Using this discovery cohort, we compared differences between benign $(n=$ 23) and malignant prostate tissue $(n=83)$ microbial profiles. We also systematically compared microbial species associations with more/less aggressive $\mathrm{PCa}$ using key clinicopathological factors known to be associated with $\mathrm{PCa}$ aggressiveness (pre-operative PSA level, Gleason score, T-stage) as well as post-operative BCR status (Table 1, Additional file 1: Fig. S1). Finally, for the species identified as differentially over/less abundant in the malignant as compared to the benign tissue samples, we performed host differential gene expression (DGE) analyses for elucidating the host transcriptional regulation in response to high $(n=42)$ vs. low $(n=41)$ species counts, followed by cell type enrichment analyses for exploring the differences in the cellular composition of the prostate tumor microenvironment (TME). Cell type enrichment analysis was also performed for comparing cellular differences between benign $(n=23)$ and malignant $(n=83)$ tissue samples.

\section{The microbial environment in primary prostate tumor tissue (radical prostatectomy specimens)}

We first investigated the microbial species that were most abundant in primary prostate tissue samples from patients with $\mathrm{PCa}$ in the discovery cohort. We included both benign $(n=23)$ and malignant $(n=83)$ prostate tissue samples in order to get a first impression of the species that were predominant in prostate tissue samples 
from our cohort of Danish men who had undergone radical prostatectomy. Using the full sample set $(n=106)$, we found that the most abundant microbial reads belonged to Enterobacter hormaechei, accounting for $23.9 \%$ of the microbial reads in all the samples (Table 2). Other highly abundant microbial reads belonged to Streptococcus pneumoniae (6.9\%), Acinetobacter baumannii (6.4\%), Mycobacterium sp. (5.8 \%), Salmonella enterica (5.4\%), Escherichia coli (3.7\%), Campylobacter jejuni (3.6\%), Clostridioides difficile (3.6\%), Mycobacterium abscessus (3.4\%), and Bacillus cereus (1.1\%) (Table 2). However, seven genera (Acinetobacter, Enterobacter, Streptococcus, Escherichia, Bacillus, and Mycobacterium) of the 10 highly abundant species that we identified (Table 2) are known to be common contaminants in sequencingbased microbiome studies [15, 40, 41]. The remaining three of the highly abundant species that we detected $(S$. enterica, $C$. jejuni, and $C$. difficile) are not known to be contaminants, and hence likely reflect the "true" prostate microbial ecosystem. While we were able to detect viral species in the prostate tissue samples, we did not observe any viruses with high relative abundances across all samples, corroborating previous research [13] and suggesting that viruses are not predominant members of the prostate microbial ecosystem.

\section{Microbial species diversity between benign and malignant $\mathrm{PCa}$}

Next, using six different measures of alpha diversity, we investigated overall differences in species richness (i.e., total number of species: Observed, Chao1, and ACE) and their evenness (i.e., relative abundances: Shannon, Simpson, and Inverse Simpson) between benign $(n=23)$ and malignant prostate tissue samples $(n=83)$ in the discovery cohort. Differences in alpha diversity between benign and malignant prostate tissue samples would indicate microbial dysbiosis that could be associated with PCa.

Visual inspection of the data suggested an overall reduction in species diversity in malignant tissue compared to benign tissue across all alpha diversity measures (Fig. 1A). A Wilcoxon rank sum test showed that these differences were also statistically significant for all the diversity measures (Additional file 1: Table S1), indicating decreased species richness and evenness in malignant tissue as compared to benign prostate tissue. A similar trend with an overall reduction in species diversity in the malignant ( $n=$ 16) compared to the benign tissue $(n=8)$ was also observed in the smaller validation cohort (Fig. 1B), although the differences were statistically significant for only Observed, ACE and Chao1 (Additional file 1: Table S1). Nevertheless, an overall reduction in the species diversity within malignant as compared to benign tissue samples indicates microbial dysbiosis associated with PCa development/progression.

\section{Differential abundance of species in benign vs. malignant prostate tissue samples}

We next performed differential abundance analysis between benign $(n=23)$ and malignant tissue $(n=83)$ samples in order to see whether any particular organism or group of organisms were under- or over-represented in PCa tissue in the discovery cohort. We used a cut-off criteria of an adjusted $p$ value less than 0.01 , a $\log _{2}$ fold change greater than $|0.58|$, and a normalized mean count greater than 10 across all samples, for assigning differentially abundant species. Using these criteria, we found the genus Shewanella to be significantly overabundant in the malignant as compared to benign prostate tissue samples. Similarly, we found four microbial organisms (including a virus) to have significantly lower abundances in the malignant tissue as compared to the

Table 2 Most abundant microbial species in the prostate tissue of patients who had PCa

\begin{tabular}{|c|c|c|c|c|}
\hline Species & $\begin{array}{l}\text { Total reads across all samples } \\
(n=106)\end{array}$ & $\begin{array}{l}\text { Relative proportions } \\
\text { (\% of all reads) }\end{array}$ & $\begin{array}{l}\text { Mean number of } \\
\text { reads per sample }\end{array}$ & Standard deviation \\
\hline Enterobacter hormaechei* & 674,201 & 23.9 & 6360.3 & 2749.8 \\
\hline Streptococcus pneumoniae* & 195,368 & 6.9 & 1843.1 & 1185.9 \\
\hline Acinetobacter baumannii* & 181,489 & 6.4 & 1712.1 & 2856.3 \\
\hline Mycobacterium sp.* & 164,496 & 5.8 & 1551.8 & 1174.1 \\
\hline Salmonella enterica & 153,667 & 5.4 & 1449.6 & 1078.6 \\
\hline Escherichia coli* & 104,243 & 3.7 & 983.42 & 913.3 \\
\hline Campylobacter jejuni & 102,543 & 3.6 & 967.3 & 515.7 \\
\hline Clostridioides difficile & 102,525 & 3.6 & 1235.2 & 4082.6 \\
\hline Mycobacterium abscessus* & 98,079 & 3.4 & 925.2 & 476.9 \\
\hline Bacillus cereus* & 33,484 & 1.1 & 1455.8 & 883.4 \\
\hline Other & $1,002,742$ & 35.6 & 9459.8 & 5067.9 \\
\hline
\end{tabular}

*Genera of these species are known to be common contaminants in sequencing based microbiome studies 


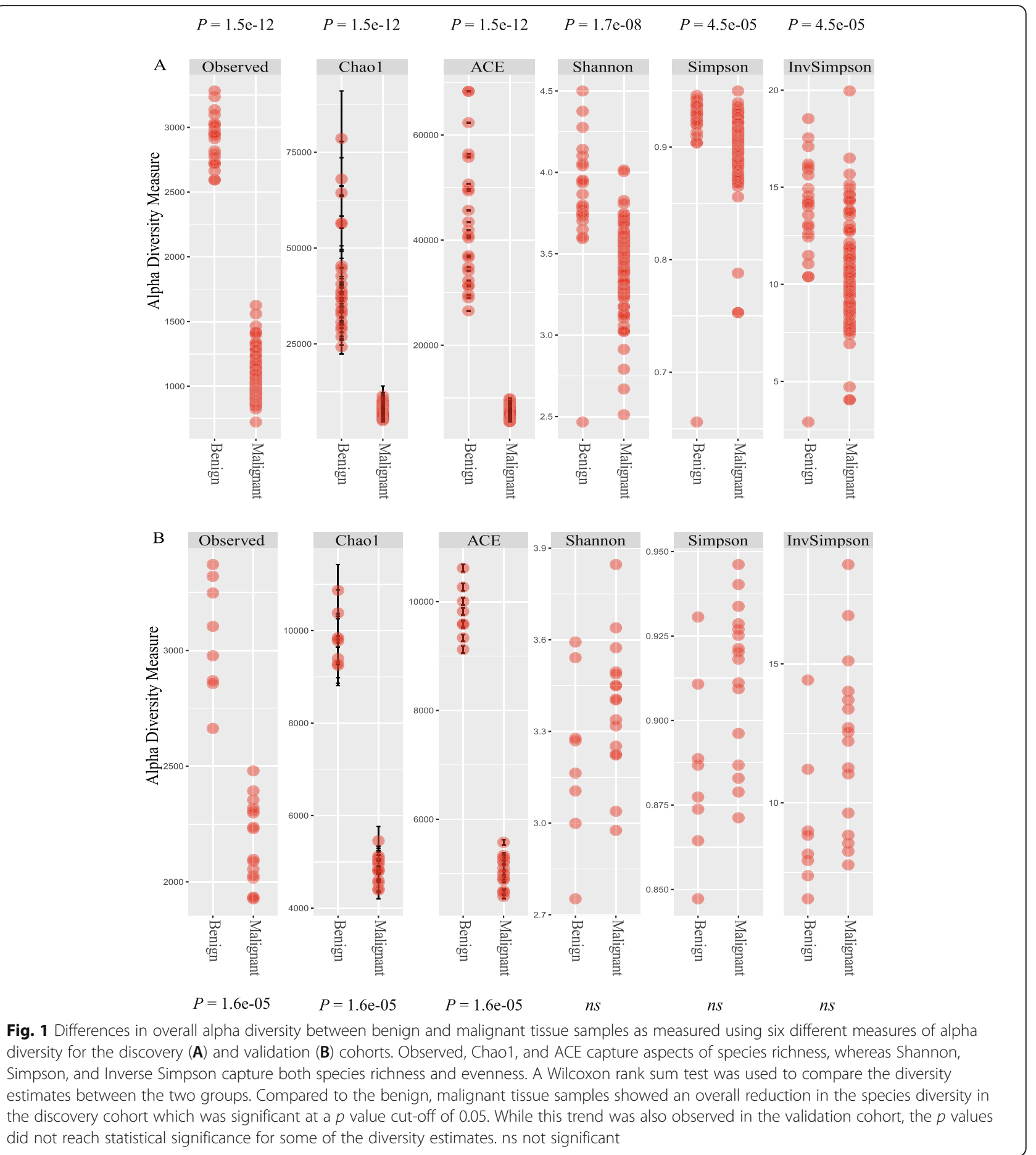

benign tissue (Fig. 2, Table 3). These included Bacteriodes fragilis, Staphylococcus saprophyticus, Vibrio parahaemolyticus, and Saimiriine betaherpesvirus.

In order to validate the association between $\mathrm{PCa}$ and the five microbial species identified as differentially abundant between benign and malignant tissue samples in the discovery cohort, we tested for their differential abundance between benign $(n=8)$ and malignant tissue
( $n=16)$ samples using an independent validation cohort (Additional file 1: Fig. S1). Of the five species identified, we were able to validate the differential abundance of three species (Shewanella, V. parahaemolyticus, and $S$. saprophyticus) at a significance cut-off adjusted $p$ value less than 0.05 and a fold change greater than 1.5. Shewanella was significantly over-abundant, whereas $V$. parahaemolyticus and $S$. saprophyticus had significantly 

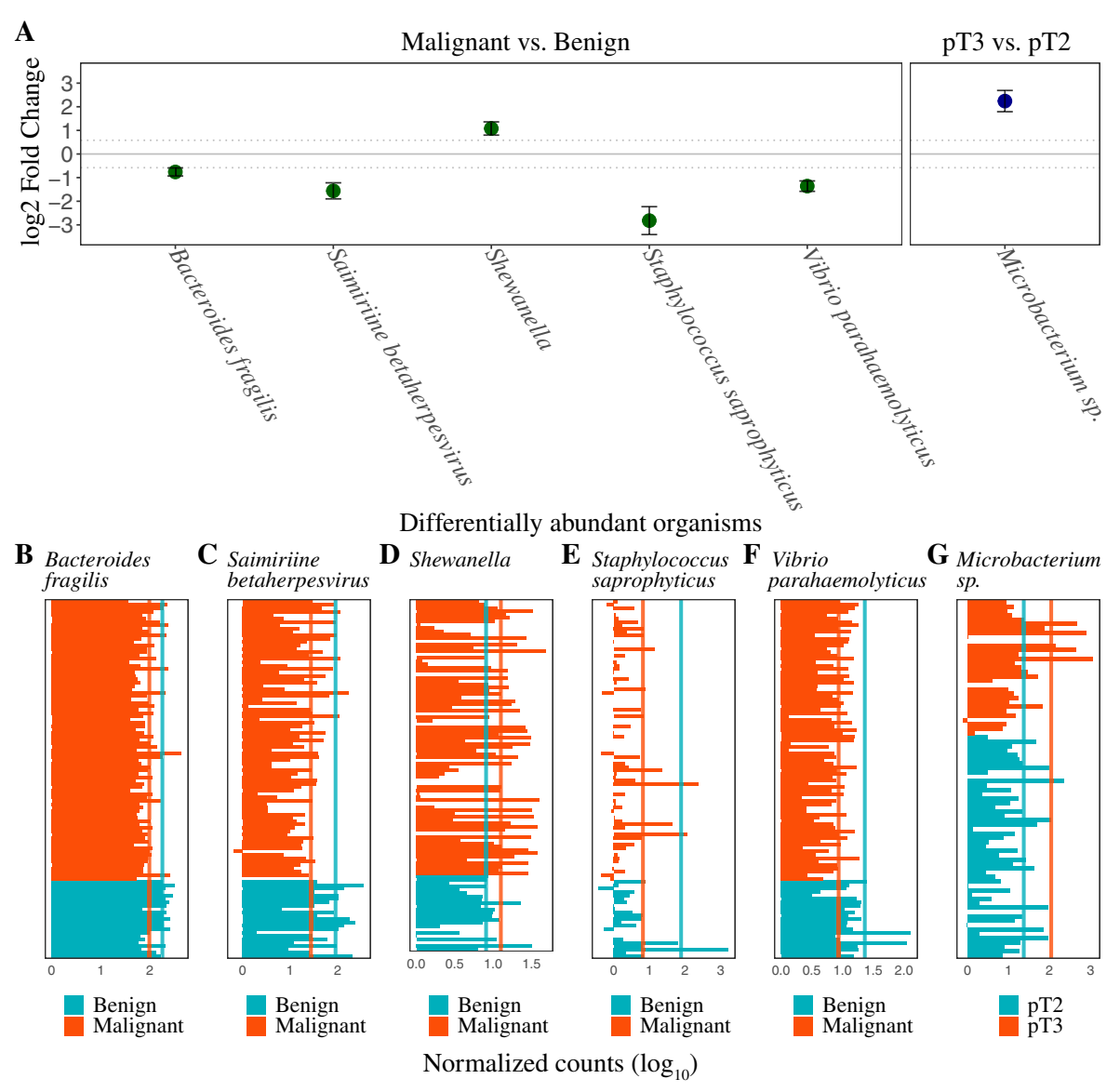

G Microbacterium

$s p$.

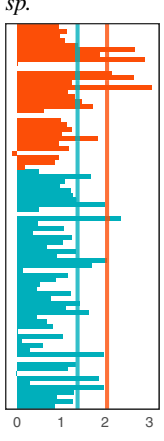

pT2

Normalized counts $\left(\log _{10}\right)$

Fig. 2 A Differentially abundant microorganisms (adjusted $p$ value $<0.01$ ) identified in the comparisons between malignant and benign tissue samples, and pathological T-stage 3 vs. 2 (pT3 vs. pT2). Error bars correspond to the standard error of the $\log _{2}$ fold change. Malignant tissue sample was associated with over-abundance of Shewanella, and under-representation of Bacteroides fragilis, Saimiriine betaherpesvirus, Staphylococcus saprophyticus, and Vibrio parahaemolyticus. Pathologically advanced T3 stage tumors were associated with significantly increased abundances of Microbacterium species. Dotted line corresponds to a $\log _{2}$ fold change cut-off value of |0.58|. B-H Normalized microbial read counts ( $x$-axis) for each patient sample (y-axis) of B. fragilis (B), S. betaherpesvirus (C), Shewanella (D), S. saprophyticus (E), V. parahaemolyticus (F), and Microbacterium sp. $(\mathbf{G})$. Values are given on a $\log _{10}$ scale. Vertical lines denote mean values. Missing bars represent a normalized count value of zero

Table 3 Differentially abundant microbial reads in various comparisons in the discovery cohort

\begin{tabular}{|c|c|c|c|c|c|c|}
\hline Organism name & Base mean & $\log _{2}$ fold change & IfcSE & Wald statistic & $p$ value & $p$ adj \\
\hline \multicolumn{7}{|c|}{ Malignant vs. benign tissue (discovery cohort) } \\
\hline Shewanella & 11.61 & 1.08 & 0.28 & 3.83 & 0.0001 & 0.0042 \\
\hline Bacteroides fragilis & 115.60 & -0.76 & 0.17 & -4.33 & $1.45 \mathrm{e}-05$ & 0.0008 \\
\hline Saimiriine betaherpesvirus & 40.72 & -1.56 & 0.34 & -4.56 & $4.99 e-06$ & 0.0004 \\
\hline Staphylococcus saprophyticus & 21.99 & -2.82 & 0.59 & -4.77 & $1.80 \mathrm{e}-06$ & 0.0002 \\
\hline Vibrio parahaemolyticus & 11.78 & -1.36 & 0.22 & -6.08 & $1.15 \mathrm{e}-09$ & $5.19 \mathrm{e}-07$ \\
\hline \multicolumn{7}{|c|}{ Pathologic T-stage 3 vs. 2 (discovery cohort) } \\
\hline Microbacterium sp. & 56.12 & 2.24 & 0.45 & 4.92 & $8.35 \mathrm{e}-07$ & 0.0049 \\
\hline
\end{tabular}

Note: Showing all differentially abundant microorganisms with an adjusted $p$ value less than 0.01 and a base mean greater than 10 . Base mean, mean of the normalized count across all samples. IfCSE standard error of the $\log _{2}$ fold change, $p$-adj Benjamini-Hochberg adjusted $p$ value 


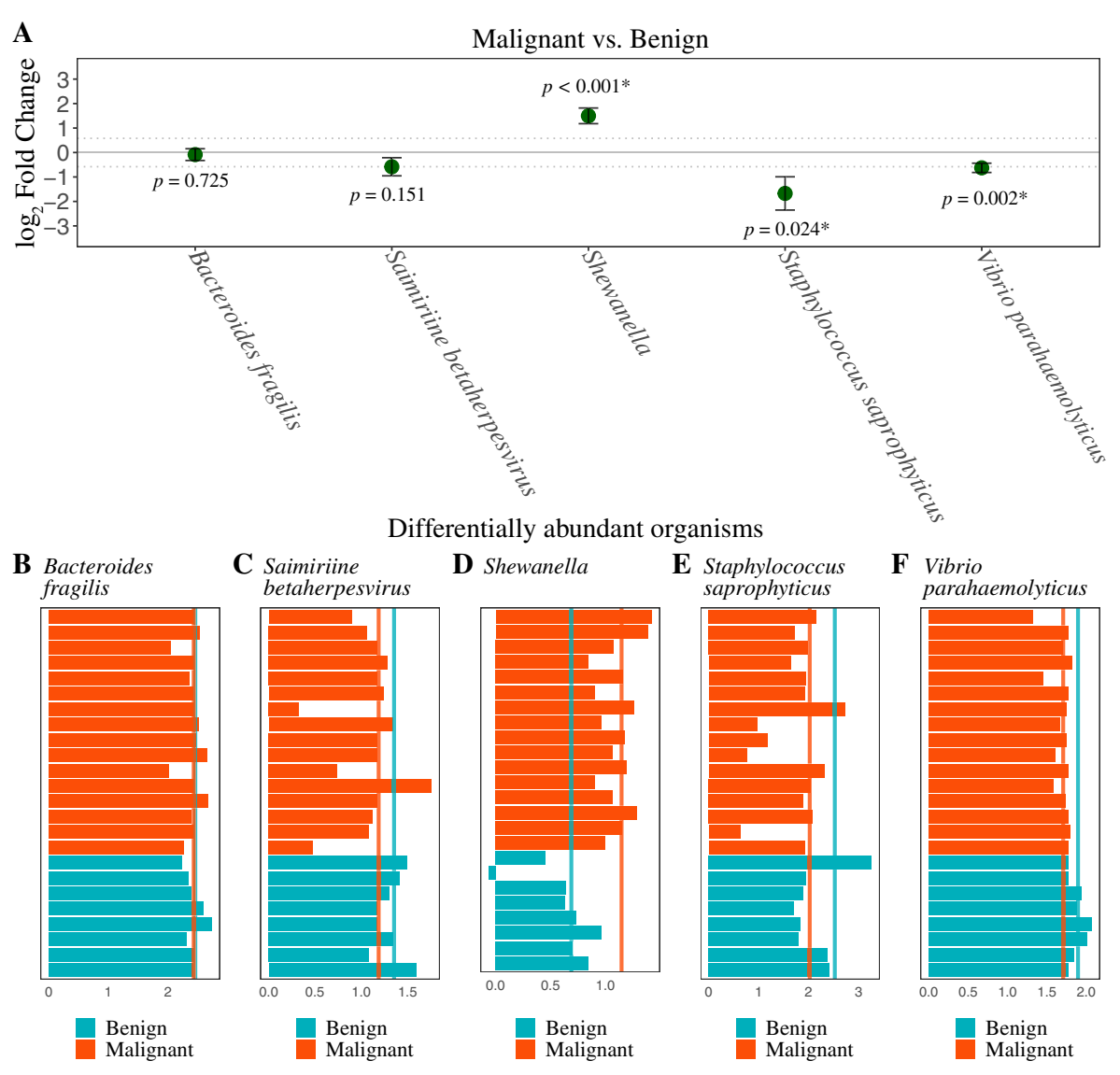

Normalized counts $\left(\log _{10}\right)$

Fig. 3 A Differentially abundant organisms between malignant and benign tissue samples identified in the discovery cohort were tested for their differential abundance in an independent validation cohort. Of the five organisms tested, three showed a significant difference in their abundance (adjusted $p$ value $<0.05$; marked by asterisk). Malignant tissue sample was associated with significant over-abundance of Shewanella, and significant under-representation of Staphylococcus saprophyticus, and Vibrio parahaemolyticus, as compared to the benign tissue samples. Bacteroides fragilis and Saimiriine betaherpesvirus showed a trend similar to that seen in the discovery cohort, although the differences did not reach statistical significance in the validation cohort. Error bars correspond to the standard error of the $\log _{2}$ fold change. Dotted line corresponds to a $\log _{2}$ fold change cut-off value of $|0.58|$. B-H Normalized microbial read counts ( $x$-axis) for each patient sample (y-axis) of B. fragilis (B), S. betaherpesvirus (C), Shewanella (D), S. saprophyticus (E), and V. parahaemolyticus (F). Values are given on a $\log _{10}$ scale. Vertical lines denote mean values

Table 4 Validation of species identified as differentially abundant between malignant and benign tissue samples in the discovery cohort

\begin{tabular}{|c|c|c|c|c|c|c|}
\hline Organism name & Base mean & $\log _{2}$ fold change & IfcSE & Wald statistic & $p$ value & $p$ adj \\
\hline \multicolumn{7}{|c|}{ Malignant vs. benign tissue (validation cohort) } \\
\hline Shewanella & 10.97 & 1.49 & 0.32 & 4.64 & $3.33 e-06$ & $1.6 \mathrm{e}-05^{*}$ \\
\hline Bacteroides fragilis & 276.86 & -0.08 & 0.24 & -0.35 & 0.7253 & 0.7253 \\
\hline Saimiriine betaherpesvirus & 17.75 & -0.58 & 0.37 & -1.54 & 0.1214 & 0.1517 \\
\hline Staphylococcus saprophyticus & 180.95 & -1.67 & 0.68 & -2.44 & 0.0145 & $0.0242^{*}$ \\
\hline Vibrio parahaemolyticus & 60.13 & -0.62 & 0.19 & -3.30 & 0.0009 & $0.0024^{*}$ \\
\hline
\end{tabular}

Note: Species identified as differentially abundant in the discovery cohort were tested for their differential abundance in the validation cohort. Significance was determined at an adjusted $p$ value less than 0.05 (marked by asterisk). Base mean, mean of the normalized count across all samples. IfcSE standard error of the $\log _{2}$ fold change, $p$-adj Benjamini-Hochberg adjusted $p$ value 
lower abundance in the malignant samples as compared to the benign samples (Fig. 3, Table 4), corroborating the results seen in the discovery cohort. Differences in $B$. fragilis and $S$. betaherpesvirus counts between benign and malignant tissue samples were not statistically significant in the smaller validation cohort but did show a trend towards lower abundance in the malignant samples, similar to that seen in the discovery cohort (Fig. 3, Table 4). The observed presence of differentially abundant (both under- and over-represented) microbial species in malignant tissue suggests to a possible microbial dysbiosis associated with PCa.

To demonstrate the validity of the microbial read processing pipeline, we performed reverse transcription PCR using species specific primers to detect $B$. fragilis in the RNA samples. We detected the presence of $B$. fragilis in all the prostate tissue samples tested ( $n=6$, Fig. 4), which was also validated by Sanger sequencing of PCR amplicons (Additional file 1: Table S2), thereby confirming the presence of the species in the tissue as opposed to errors in bioinformatics sequence annotation. Together with the detection of all five species (Shewanella, $V$. parahaemolyticus, S. saprophyticus, B. fragilis, and $S$. betaherpesvirus) in the validation cohort, these findings

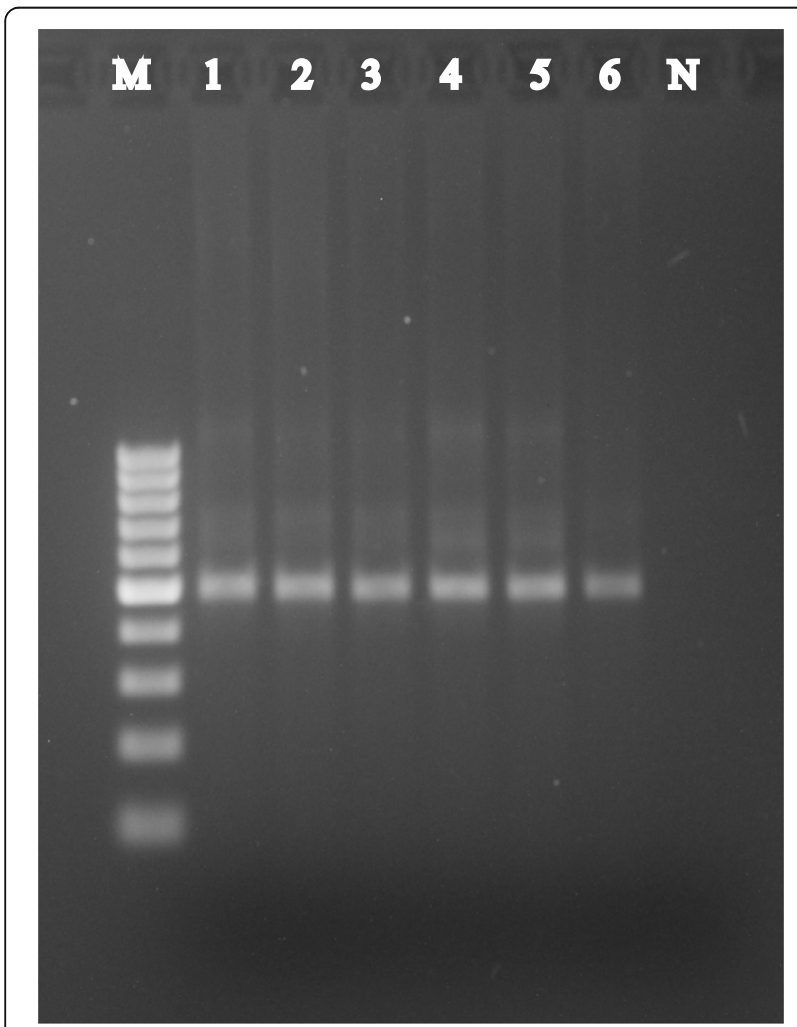

Fig. 4 Reverse transcription PCR detection of Bacteroides fragilis showing expected band around 495bp. Lanes 1, 2, and 3 are benign tissue samples. Lanes 4, 5, and 6 are malignant samples. M 100bp marker. $\mathrm{N}$ negative control support that our results are most likely to be "true" signals from the prostate tissue microbiome.

Furthermore, to test whether malignant prostate tissue samples were associated with an altered immune cell composition that might be linked with microbial dysbiosis, we performed cell type enrichment analysis in the discovery cohort comparing malignant $(n=83)$ vs. benign $(n=23)$ tissue samples. We observed significant (FDR < 0.05 ) associations between $\mathrm{PCa}$ and the presence of macrophages (including both M1 and M2 macrophages), endothelial cells, and smooth muscle cells (Fig. 5A). Macrophages had significantly higher enrichment scores in the malignant $(n=83)$ as compared to the benign $(n=$ 23) tissue samples (Fig. 5B-D), suggesting a possible link between macrophage mediated immune regulation and microbial dysbiosis in PCa.

\section{Associations between microbial presence and PCa aggressiveness}

Next, we looked for overall differences in species diversity between less vs. more aggressive PCa using alpha diversity estimates and further used differential abundance analysis to characterize microbial species that were more likely to be associated with aggressive PCa. For these analyses, we made subsets of the malignant tissue samples in the discovery cohort based on several clinicopathological factors (Table 1) including Gleason scores, pre-op PSA levels, pathologic T-stage, and BCR status. Due to the limited sample size, these analyses were not performed in the validation cohort.

For 83 malignant tissue samples, we compared samples having a low $(\leq 7, n=63)$ Gleason score with those having a high $(>7, n=20)$ Gleason score. We observed an overall increase in the species diversity in the high as compared to the low Gleason score tissue samples (Additional file 1: Fig. S2A). However, there were no differentially abundant organisms present in either low or high Gleason score groups at an adjusted $p$ value less than 0.01 , likely reflecting our stringent filtering criteria for the DGE analyses which filtered species with very low counts. Similarly, we did not find any significantly under- or over-represented species when we compared malignant tissue samples from patients having a low $(\leq 10 \mathrm{ng} / \mathrm{ml}, n=39)$ vs. high $(>10$ $\mathrm{ng} / \mathrm{ml}, n=39$ ) pre-op PSA levels, while some alpha diversity estimates suggested higher species richness in tumors from patients with higher pre-op PSA levels (Additional file 1: Fig. S2B). For the comparison between pathological T2 $(n=51)$ vs. T3 $(n=31)$ stages, we observed an overall increased species diversity in pT3 vs. pT2 (Additional file 1: Fig. S2C). Differential abundance analysis between these groups identified Microbacterium sp. to have significantly increased abundances in the more advanced pT3 stage tissue samples (adjusted $p<0.01$, Table 3 ) compared to the less advanced pT2 tissue (Fig. 2). This suggests an 


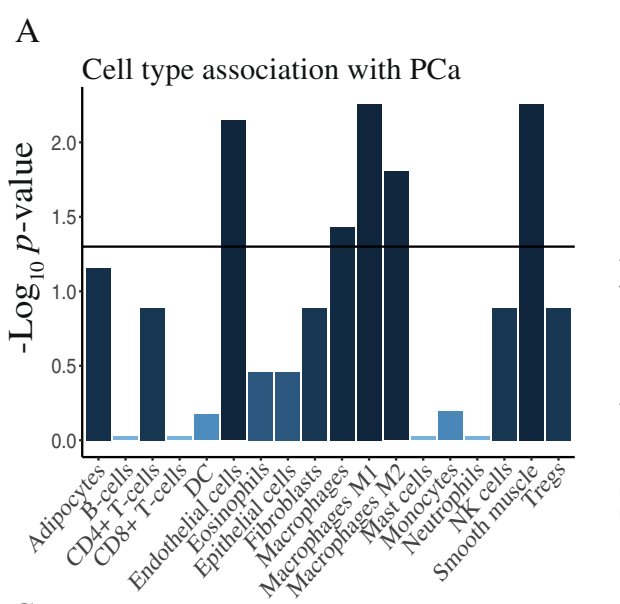

$\mathrm{C}$

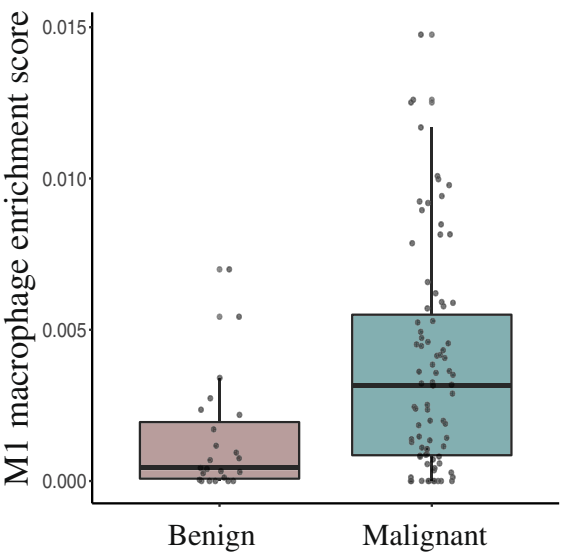

$\mathrm{E}$

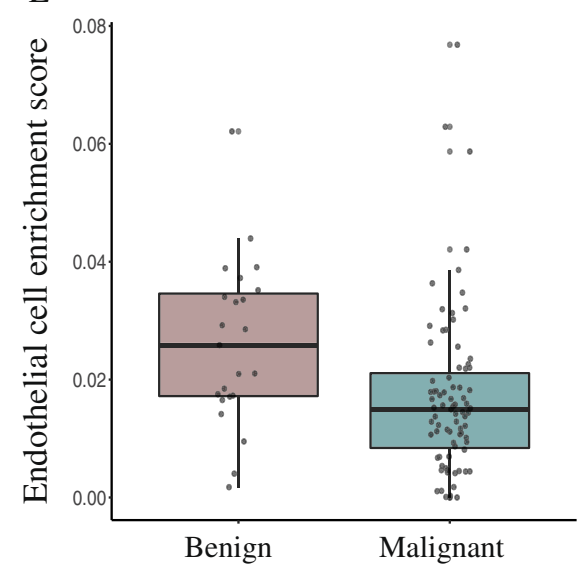

B

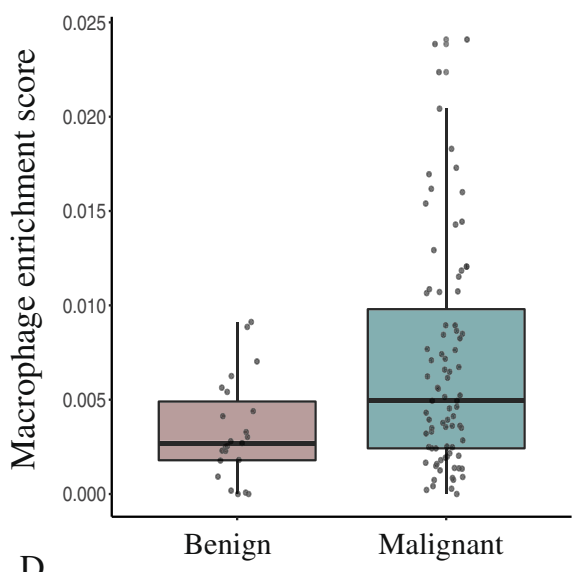

D

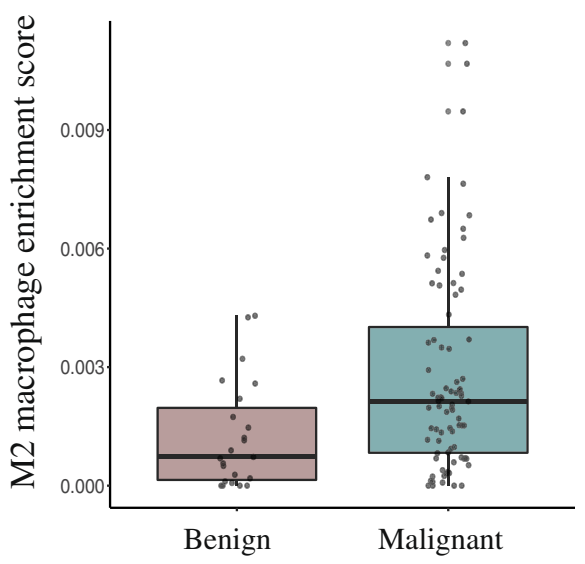

$\mathrm{F}$

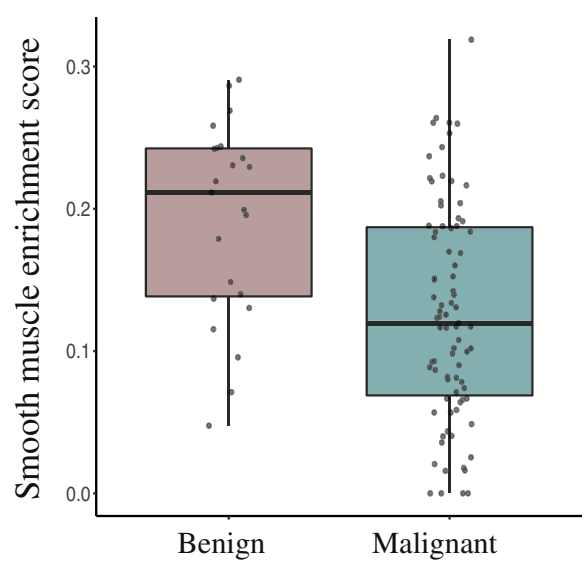

Fig. 5 A Bar chart showing association between cell types and PCa. A Wilcoxon test was used to determine significant associations. Horizontal line corresponds to a $p$ value cut-off of 0.05 . FDR corrected $p$ values are reported. Macrophages, M1 macrophages, M2 macrophages, endothelial cells, and smooth muscle showed a significant association with PCa. B-F Boxplots showing differences in the enrichment scores between benign vs. malignant tissue samples for $\mathbf{B}$ macrophages, C M1 macrophages, D M2 macrophages, E endothelial cells, and $\mathbf{F}$ smooth muscle

association between Microbacterium sp. and PCa disease progression; however, further investigations are warranted.

In order to test whether microbial dysbiosis could be associated with BCR, we compared malignant tissue samples $(n$
$=81)$ from patients in the discovery cohort who had BCR $(n$ $=28$ ) to those who did not (BCR-free; $n=53$ ). However, in this cohort, we did not find any organisms to be significantly differentially abundant in the patients who had BCR compared to those who did not, but some alpha diversity 
estimates suggested higher species richness in tumors from patients suffering BCR (Additional file 1: Fig. S2D).

\section{Host transcriptional regulation in PCa with high vs. low counts of Shewanella, Vibrio parahaemolyticus, or Staphylococcus saprophyticus}

To test whether malignant tissue samples harbouring high $(n=42)$ vs. low $(n=41)$ species counts of Shewanella, or low $(n=41)$ vs. high $(n=42)$ counts of $V$. parahaemolyticus and $S$. saprophyticus, respectively, were associated with altered host gene expression, we performed DGE analyses for the tissue samples in the discovery cohort. For the analyses comparing high vs. low Shewanella count groups, we identified 501 genes to be differentially expressed between the two groups at an adjusted $p$ value $<0.05$. Of these, 71 genes were upregulated, whereas 131 genes were downregulated in the high as compared to the low Shewanella count group at a fold change cut-off of $|1.5|$ (Fig. 6A, Additional file 2: Table S3). KEGG pathway analyses of the genes upregulated in the high vs. low Shewanella count groups showed significant (FDR $<0.05)$ enrichment of oxidative phosphorylation and metabolic pathways (Table 5), indicating increased energy utilisation by the malignant tissue samples with high abundance of Shewanella. Furthermore, genes that were downregulated were significantly enriched for Toll-like receptor signaling pathway (Table 5), indicating a downregulated immune system in prostate tumors with high abundance of Shewanella.

While no significant KEGG pathways were observed for the analyses of $S$. saprophyticus, pathways relating to olfactory transduction, retinol metabolism, steroid hormone biosynthesis, and cytochrome P450 mediated drug and xenobiotic metabolism pathways were significantly enriched in the malignant samples having low (vs. high) abundance of $V$. parahaemolyticus (Additional file 3: Table S4 and Additional file 1: Table S5). The enrichment of, e.g., drug metabolism and steroid hormone biosynthesis pathways, could suggest that patients with low $V$. parahaemolyticus counts might be more prone to developing drug resistance. Furthermore, genes involved in pathways relating to focal adhesion, mineral absorption, regulation of actin cytoskeleton, ECM-receptor interaction, vascular smooth muscle contraction, and cardiomyopathy were significantly downregulated in the malignant samples having low (vs. high) $V$. parahaemolyticus counts (Additional file 3: Table S4 and Additional file 1: Table S5). This may indicate altered regulatory interactions between the TME and PCa cells in tumors with low abundance of $V$. parahaemolyticus.
A High vs. low Shewanella abundance

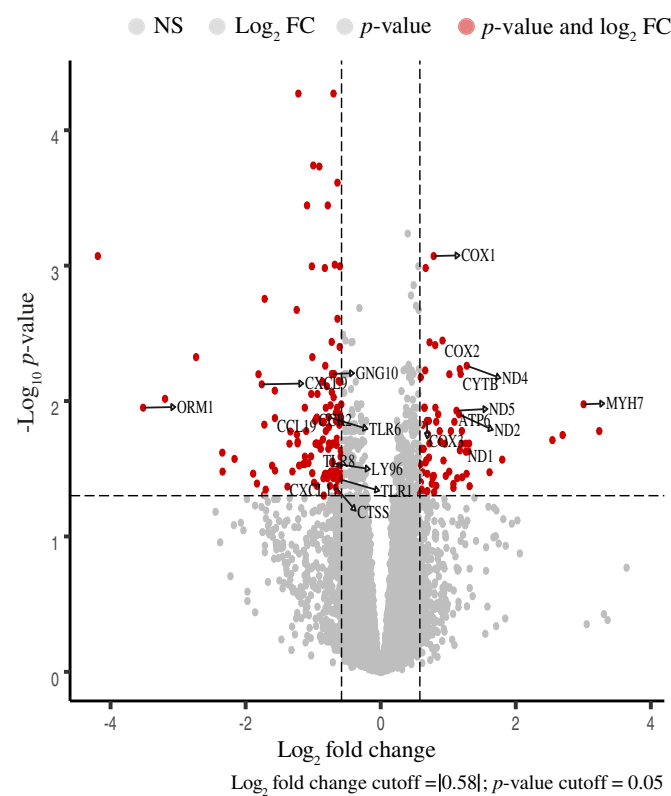

B Cell type association with Shewanella

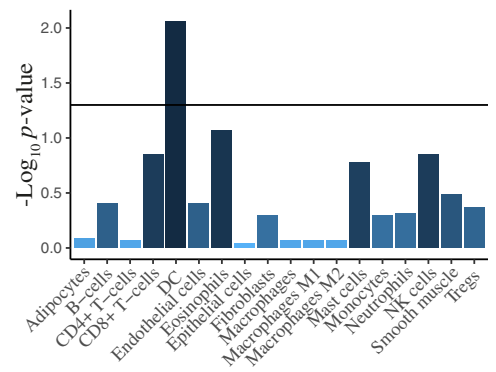

C

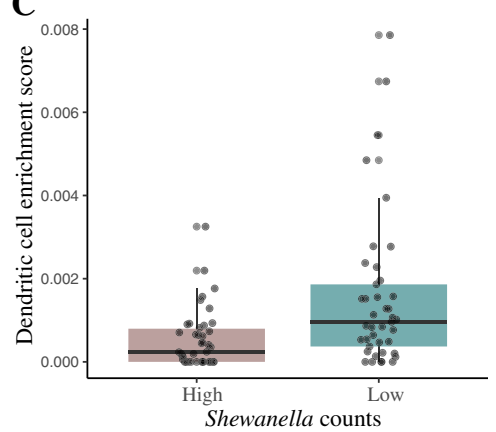

Fig. 6 A Volcano plot showing differentially expressed host genes in the comparison between high vs. low Shewanella counts group. Genes involved in significant KEGG pathways (Table 5) are also labelled in the plot. Benjamini-Hochberg corrected $p$ values are reported. NS not significant. FC fold change. B Bar chart showing association between cell types and Shewanella. A Wilcoxon test was used to determine significant associations. Horizontal line corresponds to a $p$ value cut-off of 0.05 . FDR corrected $p$ values are reported. Dendritic cells (DC) showed a significant association with Shewanella. C Boxplot showing differences in the enrichment score for DC between high vs. low Shewanella counts group 
Table 5 Results from Kyoto Encyclopedia of Genes and Genomes (KEGG) pathway analysis. Upregulated and downregulated host genes identified from the comparison between high vs. low Shewanella count group were used for KEGG pathway analysis

\begin{tabular}{|c|c|c|c|}
\hline \multicolumn{4}{|c|}{ Significant KEGG pathways in upregulated genes } \\
\hline Pathway & $\begin{array}{l}\text { Fold } \\
\text { enrichment }\end{array}$ & $p$ value & $p$ adj \\
\hline Oxidative phosphorylation & 21.88 & $\begin{array}{l}1.32 \mathrm{e} \\
-11\end{array}$ & $\begin{array}{l}4.74 e \\
-10\end{array}$ \\
\hline Parkinson's disease & 20.49 & $\begin{array}{l}2.56 e \\
-11\end{array}$ & $\begin{array}{l}4.74 e \\
-10\end{array}$ \\
\hline Metabolic pathways & 3.25 & $\begin{array}{l}1.77 e \\
-05\end{array}$ & $\begin{array}{l}2.19 e \\
-04\end{array}$ \\
\hline Cardiac muscle contraction & 17.63 & $\begin{array}{l}1.38 \mathrm{e} \\
-04\end{array}$ & 0.0012 \\
\hline Alzheimer's disease & 7.87 & 0.0029 & 0.0215 \\
\hline Huntington's disease & 6.89 & 0.0046 & 0.0289 \\
\hline \multicolumn{4}{|c|}{ Significant KEGG pathways in downregulated genes } \\
\hline Pathway & $\begin{array}{l}\text { Fold } \\
\text { enrichment }\end{array}$ & $p$ value & $p$ adj \\
\hline $\begin{array}{l}\text { Toll-like receptor signaling } \\
\text { pathway }\end{array}$ & 12.61 & $\begin{array}{l}1.31 \mathrm{e} \\
-05\end{array}$ & $\begin{array}{l}8.91 e \\
-04\end{array}$ \\
\hline
\end{tabular}

$p$-adj $p$ value corrected for false discovery rate

Host cell type enrichment in PCa with high vs. low counts of Shewanella, Vibrio parahaemolyticus, or Staphylococcus saprophyticus

Finally, to test whether malignant tissue samples harbouring high vs. low species counts of Shewanella, and low vs. high counts of $V$. parahaemolyticus and $S$. saprophyticus, respectively, were associated with an altered immune/stromal architecture, we performed cell type enrichment analysis using xCell [37]. For the comparison between high $(n=42)$ vs. low $(n=41)$ Shewanella counts group within the malignant samples, we observed a significant (FDR $<0.05)$ inverse association between Shewanella genera counts and dendritic cells (DC, Fig. 6B) with the high Shewanella count group having a lower DC enrichment score compared to the low count group (Fig. 6C). This corroborates the downregulation of immune related genes observed in the DGE analyses and indicates an important role for DCs in PCa pathophysiology.

Further, malignant samples having a low $(n=41)$ vs. high $(n=42) V$. parahaemolyticus counts had a significantly (FDR < 0.05) lower enrichment score for adipocytes, fibroblasts, and smooth muscle (Additional file 1: Fig. S3A-C) and a significantly higher enrichment score for monocytes, B cells, CD8+ T cells, mast cells, M2 macrophages, $\mathrm{T}$ regulatory cells (Additional file 1: Fig. S3D-I), and neutrophils (borderline significant, not shown), suggesting altered host immunity and dysregulated TME architecture, as also indicated by the downregulation of genes and pathways involved in, e.g., focal adhesion, regulation of actin cytoskeleton, and ECM-receptor interaction, that were identified in our DGE and KEGG pathway analyses, respectively. However, for S. saprophyticus, we did not observe any significant associations with the host stroma or immune cell types.

\section{Discussion}

Over the past decade, a more holistic approach towards understanding human diseases has led us to the holobiont nature of many diseases, including cancer. The role of the human microbiome in cancer has increasingly garnered attention from the scientific community, both in terms of its direct and indirect roles in carcinogenesis, as well as its diagnostic and prognostic potential [4244]. So far, no single pathogenic species has been causally associated with $\mathrm{PCa}$, but a growing body of evidence suggests that changes to the healthy microbiota (i.e., microbial dysbiosis) could play a major role in disease occurrence, progression, and/or treatment outcome (reviewed by 14). Thus, it is important to map the microbiota of the benign and diseased prostate tissue environment to better understand the link between $\mathrm{PCa}$ and the prostate microbiome.

A number of factors can determine the resident human microbial ecosystem, including diet, ethnicity, and geography which often distinguishes populations $[45,46]$. We here aimed to investigate the role of prostate microbiota in PCa development or progression in a cohort of Danish men and provide a comprehensive analysis of the benign (adjacent normal) and diseased (malignant) prostate tissue microbiome. Using a metatranscriptomic approach, we found several bacterial species to have very high relative abundances in the prostate tissue among Danish men who had PCa (discovery cohort). These included genera such as Enterobacter, Acinetobacter, Streptococcus, and Escherichia which have also been reported to be abundant in prostate tissue in other studies [12, 47], but are also known to be common contaminants in sequencing-based microbiome studies $[15,41]$. Thus, whether these are true representative species of the prostate microbiome or whether these are introduced during procedural handling requires further investigation.

Additionally, we identified Mycobacterium sp. and Salmonella enterica at high relative proportions in this Danish PCa discovery cohort. Of the species that have not previously been reported as contaminants in sequencing studies, we found $S$. enterica, C. jejuni, and C. difficile to have relatively high abundances in the prostate tissue of PCa patients, perhaps more accurately reflecting the "true" microbial ecology of the prostate.

While many studies have reported Propionibacterium acnes (renamed Cutibacterium acnes) to be abundant in 
prostate tissue [12, 47], $P$. acnes did not represent a major taxa across all samples in this patient cohort. Thus, our results do not indicate an association between $\mathrm{PCa}$ and the pro-inflammatory effects of $P$. acnes as suggested in other studies [11, 48, 49]. It is noteworthy that Propionibacterium is a common contaminant in sequencing studies [15, 40, 41], and most sequencing-based microbiome studies have failed to account for this possibility, perhaps explaining the contradictory results that we observed. In addition, factors such as differences in patient cohorts, sample types, sample handling, sample size, and the data analysis methodology employed can all influence which microbial species are detected, partly explaining discrepancies in the results between our study and previous studies.

\section{Microbial dysbiosis between malignant and benign tissue samples identified through differential abundance analysis}

We observed an overall reduction in the species diversity within the malignant as compared to the benign tissue samples from patients with $\mathrm{PCa}$ in both the discovery cohort from Denmark and the validation cohort from France. Corroborating these findings, our differential abundance analysis comparing malignant vs. benign tissue samples revealed four species to have significantly lower abundances in the malignant tumor tissue. Validity of these findings and of the microbial annotation pipeline was demonstrated by performing a reverse transcription PCR which successfully detected the presence of Bacteroides fragilis in all tested samples, as also confirmed by Sanger sequencing of the PCR amplicons. The presence of these species were also validated in an independent cohort in which two of the four species also showed significantly lower abundance in the malignant vs. benign tissue samples.

Interestingly, the observed lower abundances of $B$. fragilis in the malignant as compared to the benign tissue samples in the discovery cohort is in contrast to the suspected role played by this organism in colon and rectal cancers [50]. For example, rectal cancer was associated with higher abundances of $B$. fragilis in the tumor but not non-tumor tissue samples [51]. Such a result is however not surprising, given an organism can potentially have different roles in different organs. For example, increased tissue abundances of the genus Lactobacillus is reported to be associated with breast cancer [52], whereas a lower abundance of lactobacilli in the cervicovaginal microbiome is thought to be associated with ovarian cancer [53].

The only microbe showing significantly higher abundance in malignant tissue as compared to the benign tissue in the discovery cohort and validated in the independent cohort belonged to the genus Shewanella. This genus has not been reported in PCa previously, although higher abundances of Shewanella have been reported in the colon mucosa of patients with rectal and distal cancers as opposed to proximal colorectal cancer [54]. This could suggest to an association between Shewanella and at least some cancer types, making it a highly interesting candidate for further studies.

\section{Altered host immune regulation associated with increased Shewanella abundance}

Differential expression and KEGG pathway analyses between tissue samples in the discovery cohort having high vs. low Shewanella count revealed significant downregulation of key immune related genes and pathways in the high Shewanella count group, including Toll-like receptors (TLR) such as TLR1, TLR6, and TLR8. Human TLRs are expressed by leukocytes such as dendritic cells (DC) and forms part of the innate immune system that is involved in pathogen recognition [55]. Downregulation of the TLR signaling pathway in the malignant tissue samples with high Shewanella count could indicate pathogen associated immune dysregulation. However, no tissue inflammation within the prostate was evident in the histology for a majority of the patients with malignant tumor (77/83, data not shown). Thus, it may be unlikely that Shewanella induces tissue inflammatory responses that could lead to $\mathrm{PCa}$ development. It is more likely that decreased immune activity in the malignant tumor of these patients might provide a conducive tumor microenvironment for the growth of this bacteria.

Decreased enrichment scores for dendritic cells in the high vs. low Shewanella group also suggest the existence of a downregulated adaptive immune-system, since DCs have a crucial role in antigen presentation to $\mathrm{T}$ cells [56]. Decreased DC activity within these malignant tissue samples could perhaps enable immune evasion by Shewanella. However, we did not find any significant differences in other immune cell types between high vs. low Shewanella count group, which is also consistent with the absence of histologically visible tissue inflammation in most patients. While the observed link between Shewanella and DC remains to be investigated in more depth, our results suggest that presence of high levels of Shewanella in the tumor may increase vulnerability towards cancer immunotherapy that targets TLR in DCs [57]. Further characterization of the TLRs within the malignant tissue is necessary to validate whether these TLRs are in fact associated with DC.

\section{Altered host gene expression associated with decreased V. parahaemolyticus abundance}

Differential gene expression and KEGG pathway analyses between tissue samples in the discovery cohort having low vs. high $V$. parahaemolyticus count revealed significant upregulation of genes involved in 
olfactory transduction pathways in the low $V$. parahaemolyticus count group. Genes encoding twentytwo different olfactory receptors (OR) were upregulated in this group. ORs are G-protein-coupled receptors that detect odorants (chemosensation). They are mainly expressed in the olfactory epithelium, but have also been shown to be over-expressed in prostate tumor cells [58], where they could play a role in promoting cancer invasiveness and metastasis [59]. Interestingly, five of the OR genes that we identified (OR51T1, OR51S1, OR51G2, OR51A7, and OR51F2) were also significantly upregulated in abiraterone/ enzalutamide resistant VCap xenograft tumors as compared to tumors prior to castration; Pre-Cx [60]. Of note, OR51T1 and OR51S1 were also significantly upregulated in the castration resistant PCa (CRPC) vs. Pre-Cx VCap tumors in the same study. Taken together with the upregulation of genes involved in steroid hormone biosynthesis and drug metabolism pathways in low vs. high $V$. parahaemolyticus counts group as observed in our study, these findings suggest that patients with a low abundance of $V$. parahaemolyticus might be more likely to progress towards a castration resistant phenotype and/or might be more prone to developing drug resistance following treatment with abiraterone/enzalutamide.

\section{Species associated with advanced disease stage}

Comparisons of malignant tissue microbiome from patients with pathological T-stages $\mathrm{pT} 2$ and $\mathrm{pT} 3$ revealed significantly higher abundances of Microbacterium sp. in $\mathrm{pT} 3$ prostate cancer samples in the discovery cohort. Microbacterium sp. are nosocomial infectious agents that have also been reported in the blood of, e.g., lung, and pancreatic cancer patients, although disease stage of the underlying malignancy was not significantly correlated with Microbacterium $s p$. infection rate [61]. While we detected Microbacterium sp. within malignant tissue samples in the validation cohort (mean number of reads $=54.3$, standard deviation $=29.1$ ), we did not test for its differential abundance due to the limited sample size in this cohort. Thus, further validation of its association with $\mathrm{PCa}$ using a larger cohort is warranted. Whether these could be a potential novel target for therapeutic intervention in patients having more advanced pathological tumor stages also remains to be explored.

Our study has some limitations. First, we did not test for possible sources of contamination during procedural handling as all analyses were based on existing total RNAseq data. However, we took a more conservative approach and excluded samples that had very high abundances of taxa that are known to be contaminating sequences from reagent kits based on previously published studies. Additionally, by using metatranscriptomic analysis, it is possible that we missed microbial taxa that are not actively transcribing or have a low level of transcription, which could have been captured by metagenomic analysis. Our study did not yield microbial read counts in sufficient numbers to enable elucidation of the functional relevance of the prostate microbiome and its association with PCa. Finally, the lack of non-PCa control samples limits our conclusions to differences between benign (adjacent normal) and malignant tumor tissue. However, collection of truly normal prostate tissue is ethically challenging and also difficult for men in this age group (mean approx. 65 years), where occult $\mathrm{PCa}$ and/or benign prostatic hyperplasia is commonly observed [62].

\section{Conclusions}

In conclusion, we show that prostate cancer is associated with dysbiosis of the prostate microbial ecosystem with reduced overall species diversity in the malignant as compared to the benign tissue samples. Differential abundances (both higher and lower) of certain species in the malignant prostate tissue could provide diagnostic and/or prognostic potential in the future. In particular, Shewanella genera might be associated with malignant transformation of the prostate tissue that could be facilitated by a decreased host immune response. Downregulation of genes involved in TLR signaling pathway and decreased enrichment of DCs were both associated with increased Shewanella counts and could be candidate targets for future immunotherapy. Upregulation of genes involved in olfactory transduction, drug metabolism, and steroid hormone biosynthesis pathways was associated with decreased V. parahaemolyticus counts and might be indicative of patients who are more susceptible to developing treatment resistance. Microbacterium $s p$. is an interesting candidate for further investigations on its association with $\mathrm{PCa}$ aggressiveness. Our results thus support the existence of an important biological link between the prostate microbiota and PCa development/ progression. These aspects should be further investigated in future research including histologically visualizing the localization of these bacteria as well as relevant host cells (e.g., epithelial/tumor, stromal, and immune cells) within intact prostate tissue specimens using in situ detection methodologies.

\section{Abbreviations}

BCR: Biochemical recurrence; CRPC: Castration resistant prostate cancer; DC: Dendritic cells; DGE: Differential gene expression; FDR: False discovery rate; KEGG: Kyoto Encyclopedia of Genes and Genomes; OR: Olfactory receptor; PCa: Prostate cancer; Pre-op PSA: Pre-operative prostate specific antigen; RNAseq: RNA sequencing; RP: Radical prostatectomy; TLR: Toll-like receptor; TME: Tumor microenvironment 


\section{Supplementary Information}

The online version contains supplementary material available at https:/doi. org/10.1186/s13073-022-01011-3.

Additional file 1. Supplementary Tables and Figures. This file contains Tables S1, S2, S5 and Figures S1-S3.

Additional file 2: Table S3. Differentially expressed host genes identified in the malignant tissue samples having high vs. low Shewanella counts.

Additional file 3: Table S4. Differentially expressed host genes identified in the malignant tissue samples having low vs. high Vibrio parahaemolyticus and Staphylococcus saprophyticus counts, respectively.

Additional file 4: Table S6. Normalised microbial count data for the discovery cohort.

Additional file 5: Table S7. Normalised microbial count data for the validation cohort.

Additional file 6: Table S8. Normalised host gene count data for the discovery cohort - benign samples.

Additional file 7: Table S9. Normalised host gene count data for the discovery cohort - malignant samples 1-42.

Additional file 8: Table S10. Normalised host gene count data for the discovery cohort - malignant samples 43-83.

\section{Acknowledgements}

We thank the NGS team at MOMA for excellent technical support. Furthermore, the technical assistance from Mette Emdal and Derya Pero Ayyildiz is gratefully acknowledged. We thank the Danish Cancer Biobank for providing samples and information on storage and handling.

\section{Authors' contributions}

PVS and KDS designed the study. MB provided the patient samples and clinical data. BU performed the histopathological analysis. MR and JF generated the RNAseq data. JF gathered and managed the clinical data. PVS analyzed the data. PVS and KDS interpreted the data. PVS wrote the original draft. PVS and KDS revised and edited the manuscript. KDS conceived and supervised the study. The authors read and approved the final manuscript.

\section{Funding}

This study was supported by grants from the Independent Research Fund Denmark and from Aarhus University.

\section{Availability of data and materials}

As the requirement for patient consent was waived in the current study, we do not have permission to deposit the full raw and individual level clinical data in a (controlled access) repository for the discovery cohort. Instead, raw data and individual level clinical data are available from the corresponding author upon reasonable request due to Danish data protection laws. Under Danish law, access to this data will require (1) Ethical approval of the new research project, which has to be applied for to the relevant Danish authority by the data owner (corresponding author of this work, Aarhus University Hospital) on behalf of the PI/collaborator of the new project. (2) After ethical approval has been obtained, a data sharing agreement for the new project is required between the data owner (corresponding author, Aarhus University Hospital) and the $\mathrm{Pl} /$ collaborator of the project and their institutions. The processed dataset used and/or analyzed in the discovery cohort is included within the manuscript as Additional file 4: Table S6 and Additional files 6, 7 and 8: Tables 58-510, respectively. The dataset corresponding to the validation cohort is available online at Gene Expression Omnibus under the accession GSE115414 (https://www.ncbi.nlm.nih.gov/ geo/query/acc.cgi?acc=GSE115414) [17]. Processed microbial read counts for the validation cohort are available as Additional file 5: Table S7.

\section{Declarations}

Ethics approval and consent to participate

All research forming the basis of this study was conducted in accordance to relevant rules, regulations, and guidelines and conform to the principles of the Declaration of Helsinki. For samples included in the discovery cohort, written consent was obtained from all participants prior to their donation of tissue samples for a research biobank (approved by The Central Denmark Region Committees on Health Research Ethics [jr. nr. 2000-0299] and The Danish Data Protection Agency [jr. nr. 2013-41-2041 and jr. nr. 2007-580010]). The present study was approved by the Danish National Committee on Health Research Ethics [jr. nr. 1302791 and jr. nr. 1603542], who waived the requirement for patient consent, as (1) the new analyses were considered to not pose any health risk or other significant disadvantages to the participants and (2) an expert committee was established to deal with potential (although unlikely) incidental genetic findings in this new study.

\section{Consent for publication}

Not applicable

\section{Competing interests}

The authors declare that they have no competing interests.

\section{Author details}

${ }^{1}$ Department of Molecular Medicine, Aarhus University Hospital, 8200 Aarhus N, Denmark. ²Department of Clinical Medicine, Aarhus University, 8200 Aarhus N, Denmark. 'Department of Pathology, Aarhus University Hospital, 8200 Aarhus N, Denmark. ${ }^{4}$ Department of Urology, Aarhus University Hospital, 8200 Aarhus N, Denmark.

Received: 15 February 2021 Accepted: 5 January 2022

Published online: 25 January 2022

\section{References}

1. Ferlay J, Ervik M, Lam F, Colombet M, Mery L, Piñeros M, et al. Global cancer observatory: cancer today. Lyon, France: International Agency for Research on Cancer 2018 [Available from: https://gco.iarc.fr/today.

2. Carioli G, Bertuccio P, Boffetta P, Levi F, La Vecchia C, Negri E, et al. European cancer mortality predictions for the year 2020 with a focus on prostate cancer. Ann Oncol. 2020;31(5):650-8. https://doi.org/10.1016/j.a nnonc.2020.02.009.

3. Rebbeck TR. Prostate cancer genetics: variation by race, ethnicity, and geography. Semin Radiat Oncol. 2017;27(1):3-10. https://doi.org/10.1016/j. semradonc.2016.08.002.

4. Al Olama AA, Kote-Jarai Z, Berndt SI, Conti DV, Schumacher F, Han Y, et al. A meta-analysis of 87,040 individuals identifies 23 new susceptibility loci for prostate cancer. Nat Genet. 2014;46(10):1103-9. https:/doi.org/10.1038/ng.3094.

5. Cai T, Santi R, Tamanini I, Galli IC, Perletti G, Bjerklund Johansen TE, et al. Current knowledge of the potential links between inflammation and prostate cancer. Int J Mol Sci. 2019;20(15):3833.

6. Sfanos KS, Yegnasubramanian S, Nelson WG, De Marzo AM. The inflammatory microenvironment and microbiome in prostate cancer development. Nat Rev Urol. 2018;15(1):11-24. https://doi.org/10.1038/ nrurol.2017.167.

7. Blander JM, Longman RS, Iliev ID, Sonnenberg GF, Artis D. Regulation of inflammation by microbiota interactions with the host. Nat Immunol. 2017; 18(8):851-60. https://doi.org/10.1038/ni.3780.

8. Rajagopala SV, Vashee S, Oldfield LM, Suzuki Y, Venter JC, Telenti A, et al The human microbiome and cancer. Cancer Prev Res (Phila). 2017;10(4):22634. https://doi.org/10.1158/1940-6207.CAPR-16-0249.

9. Poore GD, Kopylova E, Zhu Q, Carpenter C, Fraraccio S, Wandro S, et al. Microbiome analyses of blood and tissues suggest cancer diagnostic approach. Nature. 2020;579(7800):567-74. https://doi.org/10.1038/s41586-02 0-2095-1.

10. Banerjee S, Alwine JC, Wei Z, Tian T, Shih N, Sperling C, et al. Microbiome signatures in prostate cancer. Carcinogenesis. 2019;40(6):749-64. https://doi. org/10.1093/carcin/bgz008.

11. Cavarretta I, Ferrarese R, Cazzaniga W, Saita D, Luciano R, Ceresola ER, et al. The microbiome of the prostate tumor microenvironment. Eur Urol. 2017; 72(4):625-31. https://doi.org/10.1016/j.eururo.2017.03.029.

12. Feng $Y$, Ramnarine VR, Bell $R$, Volik S, Davicioni $E$, Hayes VM, et al. Metagenomic and metatranscriptomic analysis of human prostate microbiota from patients with prostate cancer. BMC Genomics. 2019;20(1): 146. https://doi.org/10.1186/s12864-019-5457-z.

13. Feng $Y$, Jaratlerdsiri W, Patrick SM, Lyons RJ, Haynes AM, Collins CC, et al. Metagenomic analysis reveals a rich bacterial content in high-risk prostate 
tumors from African men. Prostate. 2019;79(15):1731-8. https://doi.org/10.1 002/pros.23897.

14. Wheeler KM, Liss MA. The microbiome and prostate cancer risk. Curr Urol Rep. 2019;20(10):66. https://doi.org/10.1007/s11934-019-0922-4.

15. Eisenhofer R, Minich JJ, Marotz C, Cooper A, Knight R, Weyrich LS. Contamination in low microbial biomass microbiome studies: issues and recommendations. Trends in Microbiology. 2019;27(2):105-17. https://doi. org/10.1016/j.tim.2018.11.003.

16. Pinskaya M, Saci Z, Gallopin M, Gabriel M, Nguyen HT, Firlej V, et al. Reference-free transcriptome exploration reveals novel RNAs for prostate cancer diagnosis. Life Sci Alliance. 2019;2(6):e201900449.

17. Pinskaya M, Gabriel M, Morillon A. Discovery of IncRNA biomarkers of prostate cancer. 2019. Gene Expression Omnibus. Accession Number: GSE115414. https:/www.ncbi.nlm.nih.gov/geo/query/acc.cgi?acc=GSE115414

18. Dobin A, Davis CA, Schlesinger F, Drenkow J, Zaleski C, Jha S, et al. STAR: ultrafast universal RNA-seq aligner. Bioinformatics. 2013;29(1):15-21. https://doi.org/10.1093/bioinformatics/bts635.

19. Bray NL, Pimentel H, Melsted P, Pachter L. Near-optimal probabilistic RNAseq quantification. Nat Biotechnol. 2016;34(5):525-7. https://doi.org/10.1038/ nbt.3519.

20. Soneson C, Love MI, Robinson MD. Differential analyses for RNA-seq: transcript-level estimates improve gene-level inferences. F1000Res. 2015; 4:1521.

21. Ritchie ME, Phipson B, Wu D, Hu Y, Law CW, Shi W, et al. limma powers differential expression analyses for RNA-sequencing and microarray studies. Nucleic Acids Res. 2015;43(7):e47.

22. Westreich ST, Treiber ML, Mills DA, Korf I, Lemay DG. SAMSA2: a standalone metatranscriptome analysis pipeline. BMC Bioinformatics. 2018;19(1):175. https://doi.org/10.1186/s12859-018-2189-z.

23. Quinlan AR, Hall IM. BEDTools: a flexible suite of utilities for comparing genomic features. Bioinformatics. 2010;26(6):841-2. https://doi.org/10.1093/ bioinformatics/btq033.

24. Zhang J, Kobert K, Flouri T, Stamatakis A. PEAR: a fast and accurate Illumina Paired-End reAd mergeR. Bioinformatics. 2014;30(5):614-20. https://doi.org/1 0.1093/bioinformatics/btt593.

25. Buchfink B, Xie C, Huson DH. Fast and sensitive protein alignment using DIAMOND. Nat Methods. 2015;12(1):59-60. https://doi.org/10.1038/nmeth.3176.

26. Tatusova T, Ciufo S, Fedorov B, O'Neill K, Tolstoy I. RefSeq microbial genomes database: new representation and annotation strategy. Nucleic Acids Res. 2014; 42(Database issue):D553-9. https://doi.org/10.1093/nar/gkt1274.

27. McMurdie PJ, Susan H. phyloseq: an R package for reproducible interactive analysis and graphics of microbiome census data. Plos One. 2013;8(4):e61217.

28. Oksanen J, Blanchet FG, Friendly M, Kindt R, Legendre P, McGlinn D, et al. vegan: community Ecology Package. R package version 2.5-6. 2019.

29. Moore JC. Diversity, taxonomic versus functional. In: Levin SA, editor. Encyclopedia of Biodiversity. 2nd ed. Academic Press; 2013.

30. Kim BR, Shin J, Guevarra R, Lee JH, Kim DW, Seol KH, et al. Deciphering diversity indices for a better understanding of microbial communities. J Microbiol Biotechnol. 2017;27(12):2089-93. https://doi.org/10.4014/jmb.1709. 09027.

31. Hughes JB, Hellmann JJ, Ricketts TH, Bohannan BJ. Counting the uncountable: statistical approaches to estimating microbial diversity. Appl Environ Microbiol. 2001;67(10):4399-406. https://doi.org/10.1128/AEM.67.1 0.4399-4406.2001.

32. Dunn OJ. Multiple Comparisons Among Means. J Am Stat Assoc. 1961; 56(293):52-64. https://doi.org/10.1080/01621459.1961.10482090.

33. Haegeman B, Hamelin J, Moriarty J, Neal P, Dushoff J, Weitz JS. Robust estimation of microbial diversity in theory and in practice. ISME J. 2013;7(6): 1092-101. https://doi.org/10.1038/ismej.2013.10.

34. Love Ml, Huber W, Anders S. Moderated estimation of fold change and dispersion for RNA-seq data with DESeq2. Genome Biol. 2014;15(12):550. https://doi.org/10.1186/s13059-014-0550-8.

35. Benjamini Y. Hochberg. Controlling the false discovery rate: a practical and powerful approach to multiple testing. J R Stat Soc. 1995;57(1):289-300. https://doi.org/10.1111/j.2517-6161.1995.tb02031.x.

36. Huang da W, Sherman BT, Lempicki RA. Systematic and integrative analysis of large gene lists using DAVID bioinformatics resources. Nat Protoc. 2009; 4(1):44-57. https://doi.org/10.1038/nprot.2008.211.

37. Aran $D, H u Z$, Butte AJ. xCell: digitally portraying the tissue cellular heterogeneity landscape. Genome Biol. 2017;18(1):220. https://doi.org/10.11 86/s13059-017-1349-1.
38. Matsuda K, Tsuji H, Asahara T, Matsumoto K, Takada T, Nomoto K. Establishment of an analytical system for the human fecal microbiota, based on reverse transcription-quantitative PCR targeting of multicopy rRNA molecules. Appl Environ Microbiol. 2009;75(7):1961-9. https://doi.org/10.112 8/AEM.01843-08

39. Altschul SF, Gish W, Miller W, Myers EW, Lipman DJ. Basic local alignment search tool. J Mol Biol. 1990;215(3):403-10. https://doi.org/10.1016/S0022-2 836(05)80360-2.

40. Robinson KM, Crabtree J, Mattick JS, Anderson KE, Hotopp JCD. Distinguishing potential bacteria-tumor associations from contamination in a secondary data analysis of public cancer genome sequence data. Microbiome. 2017;5(1):9. https://doi.org/10.1186/s40168-016-0224-8.

41. Salter SJ, Cox MJ, Turek EM, Calus ST, Cookson WO, Moffatt MF, et al. Reagent and laboratory contamination can critically impact sequence-based microbiome analyses. BMC Biology. 2014;12:87.

42. Zhang $H$, Sun $L$. When human cells meet bacteria: precision medicine for cancers using the microbiota. Am J Cancer Res. 2018;8(7):1157-75.

43. Xavier JB, Young VB, Skufca J, Ginty F, Testerman T, Pearson AT, et al. The cancer microbiome: distinguishing direct and indirect effects requires a systemic view. Trends Cancer. 2020;6(3):192-204. https://doi.org/10.1016/j. trecan.2020.01.004

44. Porter CM, Shrestha E, Peiffer LB, Sfanos KS. The microbiome in prostate inflammation and prostate cancer. Prostate Cancer Prostatic Dis. 2018;21(3): 345-54. https://doi.org/10.1038/s41391-018-0041-1.

45. David LA, Maurice CF, Carmody RN, Gootenberg DB, Button JE, Wolfe BE, et al. Diet rapidly and reproducibly alters the human gut microbiome. Nature. 2014;505(7484):559-63. https://doi.org/10.1038/nature12820.

46. Gupta VK, Paul S, Dutta C. Geography, Ethnicity or subsistence-specific variations in human microbiome composition and diversity. Front Microbiol. 2017;8:1162. https://doi.org/10.3389/fmicb.2017.01162.

47. Yow MA, Tabrizi SN, Severi G, Bolton DM, Pedersen J, Australian Prostate Cancer B, et al. Characterisation of microbial communities within aggressive prostate cancer tissues. Infect Agent Cancer. 2017;12(1):4. https://doi.org/1 0.1186/s13027-016-0112-7.

48. Shinohara DB, Vaghasia AM, Yu SH, Mak TN, Bruggemann H, Nelson WG, et al. A mouse model of chronic prostatic inflammation using a human prostate cancer-derived isolate of Propionibacterium acnes. Prostate. 2013; 73(9):1007-15. https://doi.org/10.1002/pros.22648.

49. Chen $Y$, Wei J. Identification of pathogen signatures in prostate cancer using RNA-seq. PLoS One. 2015;10(6):e0128955. https://doi.org/10.1371/ journal.pone.0128955.

50. Haghi F, Goli E, Mirzaei B, Zeighami H. The association between fecal enterotoxigenic B. fragilis with colorectal cancer. BMC Cancer. 2019;19(1):879.

51. Thomas AM, Jesus EC, Lopes A, Aguiar S Jr, Begnami MD, Rocha RM, et al. Tissue-associated bacterial alterations in rectal carcinoma patients revealed by 16S rRNA community profiling. Front Cell Infect Microbiol. 2016;6:179. https://doi.org/10.3389/fcimb.2016.00179.

52. Hieken TJ, Chen J, Hoskin TL, Walther-Antonio M, Johnson S, Ramaker S, et al. The microbiome of aseptically collected human breast tissue in benign and malignant disease. Sci Rep. 2016;6(1):30751. https://doi.org/10.1 038/srep30751.

53. Nené NR, Reisel D, Leimbach A, Franchi D, Jones A, Evans I, et al. Association between the cervicovaginal microbiome, BRCA1 mutation status, and risk of ovarian cancer: a case-control study. Lancet Oncol. 2019; 20(8):1171-82. https://doi.org/10.1016/S1470-2045(19)30340-7.

54. Flemer B, Lynch DB, Brown JM, Jeffery IB, Ryan FJ, Claesson MJ, et al. Tumour-associated and non-tumour-associated microbiota in colorectal cancer. Gut. 2017;66(4):633-43. https://doi.org/10.1136/gutjnl-2015-309595.

55. Kawasaki T, Kawai T. Toll-like receptor signaling pathways. Front Immunol. 2014:5:461. https://doi.org/10.3389/fimmu.2014.00461.

56. Wylie B, Macri C, Mintern JD, Waithman J. Dendritic cells and cancer: from biology to therapeutic intervention. Cancers. 2019;11(4):521.

57. Matsumoto M, Takeda Y, Tatematsu M, Seya T. Toll-like receptor 3 signal in dendritic cells benefits cancer immunotherapy. Front Immunol. 2017:8:1897. https://doi.org/10.3389/fimmu.2017.01897.

58. Weng J, Wang J, Cai Y, Stafford LJ, Mitchell D, Ittmann M, et al. Increased expression of prostate-specific G-protein-coupled receptor in human prostate intraepithelial neoplasia and prostate cancers. Int J Cancer. 2005; 113(5):811-8. https://doi.org/10.1002/ijc.20635.

59. Sanz G, Leray I, Dewaele A, Sobilo J, Lerondel S, Bouet $\mathrm{S}$, et al. Promotion of cancer cell invasiveness and metastasis emergence caused by olfactory 
receptor stimulation. PLoS One. 2014;9(1):e85110. https://doi.org/10.1371/ journal.pone.0085110.

60. Russo JW, Gao C, Bhasin SS, Voznesensky OS, Calagua C, Arai S, et al. Downregulation of dipeptidyl peptidase 4 accelerates progression to castration-resistant prostate cancer. Cancer Res. 2018;78(22):6354-62. https:// doi.org/10.1158/0008-5472.CAN-18-0687.

61. Alonso-Echanove J, Shah SS, Valenti AJ, Dirrigl SN, Carson LA, Arduino MJ, et al. Nosocomial outbreak of microbacterium species bacteremia among cancer patients. J Infect Dis. 2001;184(6):754-60. https://doi.org/10.1086/323080.

62. Zlotta AR, Egawa S, Pushkar D, Govorov A, Kimura T, Kido M, et al. Prevalence of prostate cancer on autopsy: cross-sectional study on unscreened Caucasian and Asian men. J Natl Cancer Inst. 2013;105(14): 1050-8. https://doi.org/10.1093/jnci/djt151.

\section{Publisher's Note}

Springer Nature remains neutral with regard to jurisdictional claims in published maps and institutional affiliations.

Ready to submit your research? Choose BMC and benefit from:

- fast, convenient online submission

- thorough peer review by experienced researchers in your field

- rapid publication on acceptance

- support for research data, including large and complex data types

- gold Open Access which fosters wider collaboration and increased citations

- maximum visibility for your research: over $100 \mathrm{M}$ website views per year

At BMC, research is always in progress.

Learn more biomedcentral.com/submissions 